\title{
Clinical diagnostic value and analysis of MRI combined with ultrasound in prenatal pernicious placenta previa with placenta accreta
}

\author{
Peng Guo ${ }^{1}$, Yuli Wu ${ }^{2}$, Xiaoting Yuan ${ }^{3}$, Zhenfa Wan ${ }^{4}$ \\ ${ }^{1}$ Imaging Department, Linyi Central Hospital, Linyi, China; ${ }^{2}$ Ultrasound Room of Breast Department, Linyi Central Hospital, Linyi, China; \\ ${ }^{3}$ Nephrology Department, Linyi Central Hospital, Linyi, China; ${ }^{4}$ Department of Radiology, The Fourth People's Hospital of Jinan, Jinan, China \\ Contributions: (I) Conception and design: P Guo, Y Wu; (II) Administrative support: Z Wan; (III) Provision of study materials or patients: P \\ Guo, Z Wan; (IV) Collection and assembly of data: P Guo; (V) Data analysis and interpretation: Z Wan; (VI) Manuscript writing: All authors; \\ (VII) Final approval of manuscript: All authors. \\ Correspondence to: Zhenfa Wan. Department of Radiology, The Fourth People's Hospital of Jinan, No. 50 Shifan Road, Jinan 250031, China. \\ Email: wanzhenfa123@163.com.
}

Background: Placenta previa is one dangerous disease which threatens the health of pregnant women
and their fetuses. The purpose of this study was to evaluate the clinical value of ultrasound combined with
magnetic resonance imaging (MRI) in screening for placenta previa complicated by placenta accreta.

Methods: Seventy patients with abnormal fetal position admitted to our hospital from January 2019 to January 2020 were selected for the study. Patients were diagnosed by ultrasound alone, MRI alone, and ultrasound combined with MRI. Diagnostic accuracy, sensitivity, specificity and false positive and negative diagnosis rates were evaluated against the postoperative pathological examinations of the patients.

Results: The diagnostic accuracy, sensitivity and false negative rate for ultrasound combined with MRI were $86.27 \%, 97.78 \%$ and $72.00 \%$, respectively. These results were significantly superior to those of MRI or ultrasound alone $(\mathrm{P}<0.05)$. The specificity and false positive rate for ultrasound combined with MRI were $13.73 \%$ and $5.26 \%$, respectively, which were not significantly different from those for MRI or ultrasound alone $(\mathrm{P}>0.05)$.

Conclusions: Compared with ultrasound or MRI alone, ultrasound combined with MRI has higher accuracy and sensitivity in the diagnosis of placenta previa with placenta accreta, along with lower false positive diagnosis rates. These findings are clinically important for improving the diagnostic efficiency.

Keywords: Ultrasound; magnetic resonance imaging (MRI); ultrasound combined with MRI; placenta previa; placenta accreta

Submitted Apr 20, 2021. Accepted for publication Jun 15, 2021.

doi: 10.21037/apm-21-1285

View this article at: https://dx.doi.org/10.21037/apm-21-1285

\section{Introduction}

At present, the pathogenesis of placenta previa remains unclear, and the study has indicated that the incidence of placenta previa is more than $10 \%$ (1). Some studies suggest that this condition is closely related to the age, number of previous cesarean deliveries, and smoking history of the pregnant women, while other studies have shown that patients with uterine-related diseases are more prone to placenta previa during pregnancy. During normal gestation, the villi on the placenta can interact with the villi on the endometrium to promote placenta implantation. Subsequently, the thickness of the endometrium increases, thus forming the decidua where placental villus are usually unable to invade the myometrium of the uterus (2); however, in some cases, inflammation, surgery, and dysplasia may cause endometrial damage in pregnant women and 
attachment of the embryo to these damaged sites will cause placental villi to cross the endometrium and invade the myometrium, resulting in placenta accreta (3). Clinical data has revealed that placenta accreta could lead to coagulation disorders in patients, resulting in massive and potentially fatal hemorrhage during or after delivery (4-6). Therefore, timely diagnosis of placenta previa combined with placenta accreta is of great importance during pregnancy. At present, the diagnosis of placenta previa combined with placenta accreta occurs mainly through magnetic resonance imaging (MRI) and ultrasound $(7,8)$; however, patient physique, placenta position, external environment and other factors can seriously impact diagnostic results. These issues can interfere with timely and effective diagnosis of placenta previa with placenta accreta and increase the risk of adverse events during pregnancy $(9,10)$. Therefore, it is imperative to investigate an efficient and rapid diagnostic method for placenta accreta. Some studies have confirmed that ultrasound combined with MRI can improve efficiency compared to a single diagnostic method (11). This study intends to compare the diagnostic value of ultrasound combined with MRI compared with ultrasound alone and MRI alone in the diagnosis of placenta previa with placenta accreta by evaluating the accuracy, sensitivity, specificity, false positive and negative diagnosis rates. Ultimately, these investigations aim to provide a standard reference for the clinical diagnosis of placenta previa combined with placenta accreta. We present the following article in accordance with the STARD reporting checklist (available at https://dx.doi. org/10.21037/apm-21-1285).

\section{Methods}

\section{General information}

Seventy patients admitted to our hospital from January 2019 to January 2020 with placenta previa or low-lying placenta (of varying degrees) were selected for the study. Participant age ranged from 25 to 43 years old, with an average age of $32.02 \pm 3.27$ years old.

Inclusion criteria for participants: singleton pregnancy; second or third trimester of pregnancy; no metal implants in their body; physical condition met the criteria for MRI and ultrasound; conscious with normal communication ability.

Exclusion criteria for participants: state of tension, anxiety or tachypnea; obvious fetal movements, which affected the diagnosis effects; other diseases that seriously affected the results; refusal to cooperate with the study.

The study was conducted in accordance with the Declaration of Helsinki (as revised in 2013) (12). This study was approved by the Ethics Committee of Linyi Central Hospital. Patients and their family were informed of the related contents of this study, and all subjects written informed consent.

\section{Detection methods}

Ultrasound examination: Patients were diagnosed using the GE-E8 diasonograph (GE Company, USA) and were informed to drink sufficient water to prior to examination to ensure a full bladder during imaging. Transabdominal ultrasonography and transvaginal probe were adopted with a frequency of 3.0-5.0 and 8-10 Hz, respectively. The color Doppler ultrasound was used to observe the lower segment of the uterus, including the thickness and location of the placenta, as well as the distribution and characteristics of blood flow between the posterior placental space and the patient's bladder.

MRI examination: SIEMENS Aerate 1.5T MRI scanner was used. The placenta was taken as the center for plain scanning. With the long axis of the patient's uterus as the reference, plain scanning was conducted in the transverse, sagittal and coronal planes. The scanning slice thickness and gap were 5 and $1 \mathrm{~mm}$, respectively, while the number of scanning slices was between 25 and 30 . The transverse scanning, which was perpendicular to the uterine wall, started from the uterine fundus to the pelvic floor to ensure full view of the cervix and placenta, while the sagittal and coronal scans (parallel to the long axis of the uterus) ensured full view of the uterus and cervix. Scanning sequences were as follows:

(I) In T2-weighted turbo spin-echo imaging (TSE/ T2WI) sequence, transverse scans were performed with repetition time (TR) of $3,010 \mathrm{~ms}$, echo time (TE) of $71 \mathrm{~ms}$, flip angle of $90^{\circ}$ and 2 times of radio frequency $(\mathrm{RF})$ excitation;

(II) In Haste/T2WI sequence, sagittal, transverse and coronal scans were performed with scanning parameters TR of 1,350 ms, TE of $91 \mathrm{~ms}$, flip angle of $90^{\circ}$ and 2 times of RF excitation;

(III) In True-Fisp sequence, sagittal, coronal and transverse scans were performed with scanning parameters TR of $3.8 \mathrm{~ms}$ and TE of $1.9 \mathrm{~ms}$;

(IV) In volumetric interpolated breath-hold T1weighted (VIBE-T1WI) sequence, transverse scans 
Table 1 Calculation formula of diagnostic efficiency

Accuracy rates $=(T P+T N) /(T P+T N+F P+F N) \times 100 \%$

True positive rates $=\mathrm{TP} /(\mathrm{TP}+\mathrm{FN}) \times 100 \%$

True negative rates $=\mathrm{NP} /(\mathrm{FP}+\mathrm{TN}) \times 100 \%$

False positive diagnosis rate $=\mathrm{FP} /(\mathrm{TP}+\mathrm{FP}) \times 100 \%$

False negative diagnosis rate $=\mathrm{FN} /(\mathrm{TN}+\mathrm{FN}) \times 100 \%$

TP, true positive; TN, true negative; FP, false positive; FN, false negative.

Table 2 Gold standard evaluation of different detection methods

\begin{tabular}{|c|c|c|c|c|c|c|c|c|c|c|c|}
\hline Ultrasound & \multicolumn{3}{|c|}{ Pathological examination } & MRI & \multicolumn{3}{|c|}{ Pathological examination } & $\begin{array}{l}\text { Ultrasound + } \\
\qquad \text { MRI }\end{array}$ & \multicolumn{3}{|c|}{ Pathological examination } \\
\hline$P$ & 35 & 8 & 43 & $P$ & 33 & 9 & 42 & $P$ & 44 & 7 & 51 \\
\hline $\mathrm{N}$ & 10 & 17 & 27 & $\mathrm{~N}$ & 12 & 16 & 28 & $\mathrm{~N}$ & 1 & 18 & 19 \\
\hline S & 45 & 25 & 70 & S & 45 & 25 & 70 & S & 45 & 25 & 70 \\
\hline
\end{tabular}

$\mathrm{P}$, positive; $\mathrm{N}$, negative; $\mathrm{S}$, summation; MRI, magnetic resonance imaging.

were conducted with scanning parameters TR of $7.5 \mathrm{~ms}$ and TE of $2.4 \mathrm{~ms}$. The slice thickness and gap were 3 and $1 \mathrm{~mm}$, respectively;

(V) In diffusion-weighted imaging (DWI) sequence, transverse and sagittal scans were performed with b values of 600 and $800 \mathrm{~s} / \mathrm{mm}^{2}$, TR of $2,800 \mathrm{~ms}$, TE of $80 \mathrm{~ms}$, field-of-view (FOV) $350 \times 330$, and bandwidth of 1,710 .

\section{Calculation methods}

The results of pathological examination were treated as the gold standard (Table 1).

\section{Statistical analysis}

SPSS 22.0 software was used to analyze data. Measurement data were expressed as mean value \pm standard deviation and tested using a $t$-test. Enumeration data were tested using the chi-square test. Results with $\mathrm{P}<0.05$ were considered to be statistically significant.

\section{Results}

\section{Different diagnostic methods}

Pathological examination confirmed that 45 of the 70 participants were diagnosed as implantable types (true positive) and 25 as non-implantable types (true negative). From ultrasound examination, 43 patients were classified as placenta previa complicated with placenta accreta, while 27 as placenta previa. From MRI examination, 42 patients were classified as placenta previa complicated with placenta accreta, while 28 as placenta previa. From combined MRI and ultrasound diagnosis, 51 patients were classified as placenta previa complicated with placenta accreta, while 19 as placenta previa. There were some deviations in the pathological examination results among the three diagnostic methods (Table 2).

\section{Comparison of single ultrasound and combined diagnosis}

Pathological examination results were used as the gold standard to compare the efficacy of ultrasound alone and combined ultrasound and MRI for diagnosis of placenta previa complicated with placenta accreta (Figure 1, Table 3). The results showed that the diagnostic accuracies for single ultrasound versus combined ultrasound and MRI were $81.40 \%$ and $86.27 \%$, respectively $(\mathrm{P}<0.05)$. Sensitivity for each diagnostic method was $77.78 \%$ and $97.78 \%$, respectively $(\mathrm{P}<0.05)$ and specificity was $68.00 \%$ and $72.00 \%$, respectively $(\mathrm{P}>0.05)$. False positive diagnosis rates were $18.60 \%$ and $13.73 \%$, for each method respectively $(\mathrm{P}>0.05)$ while the false negative diagnosis rates were $37.04 \%$ and $5.26 \%$, respectively $(\mathrm{P}<0.05)$. 


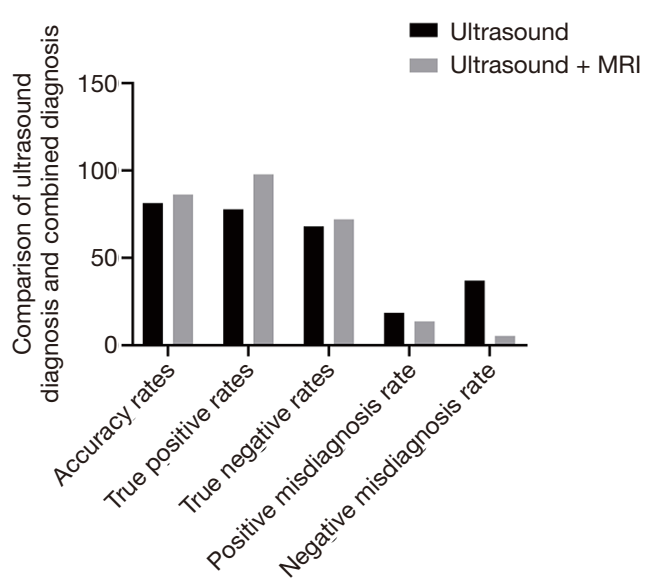

Figure 1 Comparison of ultrasound and ultrasound + MRI diagnosis. The accuracy rate, true positive rate, true negative rate, false positive diagnosis rate and false negative diagnosis rate of ultrasound were $81.40 \%, 77.78 \%, 68.00 \%, 18.60 \%$ and $37.04 \%$, while those indexes of combined diagnosis were $86.27 \%, 97.78 \%$, $72.00 \%, 13.73 \%$ and $5.26 \%$. MRI, magnetic resonance imaging.

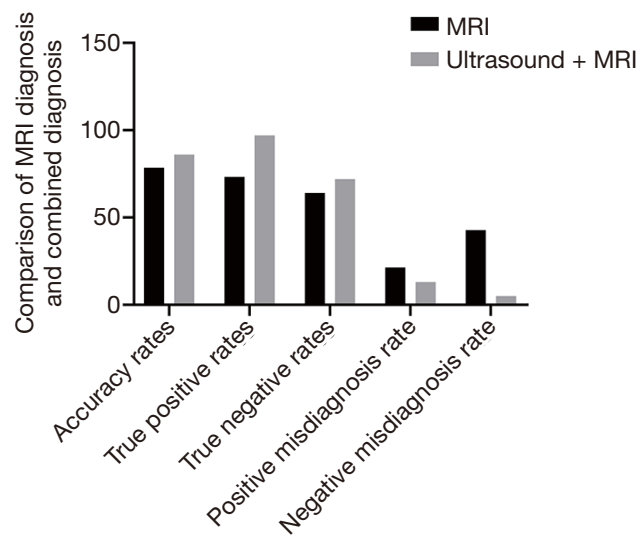

Figure 2 Comparison of MRI and ultrasound + MRI diagnosis. The accuracy rate, true positive rate, true negative rate, false positive diagnosis rate and false negative diagnosis rate of MRI were $77.78 \%, 73.33 \%, 64.00 \%, 21.43 \%$ and $42.86 \%$, respectively, while those indexes of combined diagnosis were $86.27 \%, 97.78 \%$, $72.00 \%, 13.73 \%$ and $5.26 \%$, respectively. MRI, magnetic resonance imaging.

Table 3 Comparison of ultrasound and ultrasound combined MRI diagnosis

\begin{tabular}{lccccc}
\hline Imaging methods & $\begin{array}{c}\text { Accuracy rates } \\
(\%)\end{array}$ & $\begin{array}{c}\text { True positive rates } \\
(\%)\end{array}$ & $\begin{array}{c}\text { True negative rates } \\
(\%)\end{array}$ & $\begin{array}{c}\text { Positive misdiagnosis rate } \\
(\%)\end{array}$ & $\begin{array}{c}\text { Negative misdiagnosis rate } \\
(\%)\end{array}$ \\
\hline Ultrasound & 74.29 & 77.78 & 68.00 & 11.43 & 14.29 \\
Ultrasound + MRI & 88.57 & 97.78 & 72.00 & 10.00 & 1.43 \\
$\chi^{2}$ & 6.74 & 18.65 & 0.381 & 0.107 & 11.42 \\
P value & $<0.01$ & $<0.01$ & $>0.05$ & $>0.05$ & $<0.01$
\end{tabular}

MRI, magnetic resonance imaging.

\section{Comparison of single MRI and combined diagnosis}

Pathological examination results were used as the gold standard to compare the efficacy of MRI alone and combined MRI and ultrasound for diagnosis of placenta previa complicated with placenta accreta (Figure 2, Table 4). The results showed that the diagnostic accuracies for MRI alone versus combined MRI and ultrasound were $77.78 \%$ and $86.27 \%$, respectively $(\mathrm{P}<0.05)$. Sensitivity for each diagnostic method was $73.33 \%$ and $97.78 \%$, respectively $(\mathrm{P}<0.05)$ and specificity was $64.00 \%$ and $72.00 \%$, respectively $(\mathrm{P}>0.05)$. False positive diagnosis rates were $21.43 \%$ and $13.73 \%$, respectively $(\mathrm{P}>0.05)$ while false negative diagnosis rates were $42.86 \%$ and $5.26 \%$, respectively $(\mathrm{P}<0.05)$.

\section{Discussion}

Clinical data has shown that placenta previa complicated with placenta accreta may lead to coagulation disorders, resulting in massive and potentially fatal bleeding during or after delivery $(13,14)$. Other studies have also shown that patients with uterine-related diseases are more likely to develop placenta accreta during pregnancy $(15,16)$. During pregnancy, the villi on the placenta can interact with the villi on the endometrium and promote placenta accreta. Recently, both MRI and ultrasound are major methods for diagnosing placenta previa combined with placenta accreta; however, these methods have certain deficiencies pertaining to diagnostic sensitivity and specificity $(17,18)$. This study adopted the diagnostic methods of MRI, ultrasound, and 
Table 4 Comparison of MRI and combined diagnosis

\begin{tabular}{lccccc}
\hline Imaging methods & $\begin{array}{c}\text { Accuracy rates } \\
(\%)\end{array}$ & $\begin{array}{c}\text { True positive rates } \\
(\%)\end{array}$ & $\begin{array}{c}\text { True negative rates } \\
(\%)\end{array}$ & $\begin{array}{c}\text { Positive misdiagnosis rate } \\
(\%)\end{array}$ & $\begin{array}{c}\text { Negative misdiagnosis rate } \\
(\%)\end{array}$ \\
\hline MRI & 70.00 & 73.33 & 64.00 & 12.90 & 17.10 \\
Ultrasound + MRI & 88.57 & 97.78 & 72.00 & 10.00 & 1.43 \\
$\chi^{2}$ & 10.50 & 24.19 & 1.47 & 0.41 & 14.60 \\
$\mathrm{P}$ value & $<0.01$ & $<0.01$ & $>0.05$ & $>0.05$ & $<0.01$ \\
\hline
\end{tabular}

MRI, magnetic resonance imaging.

ultrasound combined with MRI, to diagnose the presence of placenta previa complicated with placenta accreta in 70 patients with placenta previa. The clinical diagnostic value of MRI combined with ultrasound was evaluated using measures of accuracy, sensitivity, specificity, false positive and false negative diagnosis rates. From ultrasound examination, 43 patients were classified as placenta previa complicated with placenta accreta, while 27 as placenta previa. From MRI examination, 42 patients were classified as placenta previa complicated with placenta accreta, while 28 as placenta previa. From combined MRI and ultrasound diagnosis, 51 patients were classified as placenta previa complicated with placenta accreta, while 19 as placenta previa. There were some deviations in the pathological examination results among the three diagnostic methods.

Accurate diagnosis of patients with placenta accreta can reduce the risk of hemorrhage during childbirth. Some studies have pointed out that misdiagnosis and missed diagnosis can occur when ultrasound and MRI diagnosis are used in isolation $(19,20)$. Einerson et al. (21) found that the misdiagnosis rate of placenta accreta using MRI was $23 \%$ and the missed diagnosis rate was approximately $14 \%$; additionally, only two out of 14 patients with placenta accreta detected by ultrasound were confirmed using MRI. Some studies have also shown that missed diagnosis that occurs when MRI or ultrasound are used in isolation may be related to changes in bladder filling due to inflammation, the thickness of abdominal fat layer, and placenta position (22-24). Xia et al. (25) found that combined ultrasound and MRI had higher sensitivity for placenta accreta diagnosis in the second trimester of pregnancy than ultrasound or MRI alone. This study also revealed that the combined ultrasound and MRI had significantly higher specificity and accuracy for the placenta accreta diagnosis either modality alone, reducing the risk of missed diagnosis and it implications.

The results of our study showed that the diagnostic accuracies for single ultrasound versus combined ultrasound and MRI were $81.40 \%$ and $86.27 \%$, respectively $(\mathrm{P}<0.05)$. Sensitivity for each diagnostic method was $77.78 \%$ and $97.78 \%$, respectively $(\mathrm{P}<0.05)$ and specificity was $68.00 \%$ and $72.00 \%$, respectively $(\mathrm{P}>0.05)$. False positive diagnosis rates were $18.60 \%$ and $13.73 \%$, for each method respectively $(\mathrm{P}>0.05)$ while the false negative diagnosis rates were $37.04 \%$ and $5.26 \%$, respectively $(\mathrm{P}<0.05)$. In addition, our results showed that the diagnostic accuracies for MRI alone versus combined MRI and ultrasound were $77.78 \%$ and $86.27 \%$, respectively $(\mathrm{P}<0.05)$. Sensitivity for each diagnostic method was $73.33 \%$ and $97.78 \%$, respectively $(\mathrm{P}<0.05)$ and specificity was $64.00 \%$ and $72.00 \%$, respectively $(\mathrm{P}>0.05)$. False positive diagnosis rates were $21.43 \%$ and $13.73 \%$, respectively $(\mathrm{P}>0.05)$ while false negative diagnosis rates were $42.86 \%$ and $5.26 \%$, respectively $(\mathrm{P}<0.05)$.

The false negative diagnosis rate for combined ultrasound and MRI diagnosis was significantly lower than that of MRI or ultrasound diagnosis alone, which suggests combining these methods could reduce the probability of missed diagnosis and allow for improved patient management and reduce potentially avoidable adverse events.

In conclusion, compared with single ultrasound or MRI, ultrasound combined with MRI has higher accuracy and sensitivity in the diagnosis of placenta accreta in patients with placenta previa, and has a lower rate of false negative diagnosis. These results have important clinical implications for the diagnosis and timely management of this condition.

\section{Acknowledgments}

Funding: None.

\section{Footnote}

Reporting Checklist: The authors have completed the STARD reporting checklist. Available at https://dx.doi. 
org/10.21037/apm-21-1285

Data Sharing Statement: Available at https://dx.doi. org/10.21037/apm-21-1285

Conflicts of Interest: All authors have completed the ICMJE uniform disclosure form (available at https://dx.doi. org/10.21037/apm-21-1285). The authors have no conflicts of interest to declare.

Ethical Statement: The authors are accountable for all aspects of the work in ensuring that questions related to the accuracy or integrity of any part of the work are appropriately investigated and resolved. The study was conducted in accordance with the Declaration of Helsinki (as revised in 2013). This study was approved by the Ethics Committee of Linyi Central Hospital. Patients and their family were informed of the related contents of this study, and all subjects written informed consent.

Open Access Statement: This is an Open Access article distributed in accordance with the Creative Commons Attribution-NonCommercial-NoDerivs 4.0 International License (CC BY-NC-ND 4.0), which permits the noncommercial replication and distribution of the article with the strict proviso that no changes or edits are made and the original work is properly cited (including links to both the formal publication through the relevant DOI and the license). See: https://creativecommons.org/licenses/by-nc-nd/4.0/.

\section{References}

1. Iacovelli A, Liberati M, Khalil A, et al. Risk factors for abnormally invasive placenta: a systematic review and meta-analysis. J Matern Fetal Neonatal Med 2020;33:471-81.

2. Sheibak N, Mahmoudzadeh-Sagheb H, Moudi B, et al. Elevated immunoexpression of interferon-gamma in placenta tissue samples from pregnancies complicated with preeclampsia compared to the placenta previa. Pregnancy Hypertens 2020;22:175-80.

3. Yu FNY, Leung KY. Antenatal diagnosis of placenta accreta spectrum (PAS) disorders. Best Pract Res Clin Obstet Gynaecol 2021;72:13-24.

4. Melcer Y, Jauniaux E, Maymon S, et al. Impact of targeted scanning protocols on perinatal outcomes in pregnancies at risk of placenta accreta spectrum or vasa previa. Am J Obstet Gynecol 2018;218:443.e1-8.
5. Carusi DA. The placenta accreta spectrum: epidemiology and risk factors. Clin Obstet Gynecol 2018;61:733-42.

6. Duzyj CM, Cooper A, Mhatre M, et al. Placenta accreta: a spectrum of predictable risk, diagnosis, and morbidity. Am J Perinatol 2019;36:1031-8.

7. Ishibashi $\mathrm{H}$, Miyamoto $\mathrm{M}$, Shinmoto $\mathrm{H}$, et al. Response to Correspondence: Marginal sinus placenta previa is a different entity in placenta previa: a retrospective study using magnetic resonance imaging. Taiwan J Obstet Gynecol 2018;57:910.

8. Su C, Chen D. Using a cervical ripening balloon to penetrate the placenta and quickly reduce bleeding by pressing against the placenta during pregnancy termination for patients with placenta previa in the second trimester: two cases report. Medicine (Baltimore) 2020;99:e22499.

9. Chen X, Shan R, Zhao L, et al. Invasive placenta previa: Placental bulge with distorted uterine outline and uterine serosal hypervascularity at $1.5 \mathrm{~T}$ MRI - useful features for differentiating placenta percreta from placenta accreta. Eur Radiol 2018;28:708-17.

10. Kingdom JC, Hobson SR, Murji A, et al. Minimizing surgical blood loss at cesarean hysterectomy for placenta previa with evidence of placenta increta or placenta percreta: the state of play in 2020. Am J Obstet Gynecol 2020;223:322-9.

11. Jha $\mathrm{P}$, Behr S, Morgan T, et al. Imaging findings of concealed intra-amniotic hemorrhage in the setting of placenta previa and placenta accreta spectrum disorder. Emerg Radiol 2018;25:553-6.

12. World Medical Association. World Medical Association Declaration of Helsinki: ethical principles for medical research involving human subjects. JAMA 2013;310:2191-4.

13. Bourgioti C, Zafeiropoulou K, Fotopoulos S, et al. MRI prognosticators for adverse maternal and neonatal clinical outcome in patients at high risk for placenta accreta spectrum (PAS) disorders. J Magn Reson Imaging 2019;50:602-18.

14. Soyama H, Miyamoto M, Sasa H, et al. Pregnancy with "mimicking placenta previa and large cervical leiomyoma" caused by incarcerated retroverted uterus. Taiwan J Obstet Gynecol 2018;57:616-7.

15. Millischer AE, Deloison B, Silvera S, et al. Dynamic contrast enhanced MRI of the placenta: a tool for prenatal diagnosis of placenta accreta? Placenta 2017;53:40-7.

16. Chen YF, Ismail H, Chou MM, et al. Exaggerated placenta site in placenta previa: an imaging differential diagnosis of placenta accreta, placental site trophoblastic tumor and 
molar pregnancy. Taiwan J Obstet Gynecol 2012;51:440-2.

17. Bourgioti C, Zafeiropoulou K, Fotopoulos S, et al. MRI features predictive of invasive placenta with extrauterine spread in high-risk gravid patients: a prospective evaluation. AJR Am J Roentgenol 2018;211:701-11.

18. Budorick NE, Figueroa R, Vizcarra M, et al. Another look at ultrasound and magnetic resonance imaging for diagnosis of placenta accreta. J Matern Fetal Neonatal Med 2017;30:2422-7.

19. Rekawek P, Liu L, Pan S, et al. The clinical utility of magnetic resonance imaging as an adjunct to ultrasound in the diagnosis of placenta accreta spectrum disorders. J Matern Fetal Neonatal Med 2021. [Epub ahead of print]. doi: 10.1080/14767058.2021.1903861.

20. Chu C, Zhao S, Ding M, et al. Combining clinical characteristics and specific magnetic resonance imaging features to predict placenta accreta. J Comput Assist Tomogr 2019;43:775-9.

21. Einerson BD, Nelson RE, Sandoval G, et al. Cost

Cite this article as: Guo P, Wu Y, Yuan X, Wan Z. Clinical diagnostic value and analysis of MRI combined with ultrasound in prenatal pernicious placenta previa with placenta accreta. Ann Palliat Med 2021;10(6):6753-6759. doi: 10.21037/apm-211285 of elective labor induction compared with expectant management in nulliparous women. Obstet Gynecol 2020;136:19-25.

22. Kilcoyne A, Shenoy-Bhangle AS, Roberts DJ, et al. MRI of placenta accreta, placenta increta, and placenta percreta: pearls and pitfalls. AJR Am J Roentgenol 2017;208:214-21.

23. Kumar I, Verma A, Ojha R, et al. Invasive placental disorders: a prospective US and MRI comparative analysis. Acta Radiol 2017;58:121-8.

24. Ueno Y, Maeda T, Tanaka U, et al. Evaluation of interobserver variability and diagnostic performance of developed MRI-based radiological scoring system for invasive placenta previa. J Magn Reson Imaging 2016;44:573-83.

25. Xia H, Ke SC, Qian RR, et al. Comparison between abdominal ultrasound and nuclear magnetic resonance imaging detection of placenta accreta in the second and third trimester of pregnancy. Medicine (Baltimore) 2020;99:e17908. 